\title{
ACTUATOR FAULT TOLERANCE IN CONTROL SYSTEMS WITH PREDICTIVE CONSTRAINED SET-POINT OPTIMIZERS
}

\author{
PIOTR M. MARUSAK, PIOTR TATJEWSKI \\ Institute of Control and Computation Engineering \\ Warsaw University of Technology, ul. Nowowiejska 15/19, 00-665 Warsaw, Poland \\ e-mail: \{Marusak, Tatjewski\}@ia.pw.edu.pl
}

\begin{abstract}
Mechanisms of fault tolerance to actuator faults in a control structure with a predictive constrained set-point optimizer are proposed. The structure considered consists of a basic feedback control layer and a local supervisory set-point optimizer which executes as frequently as the feedback controllers do with the aim to recalculate the set-points both for constraint feasibility and economic performance. The main goal of the presented reconfiguration mechanisms activated in response to an actuator blockade is to continue the operation of the control system with the fault, until it is fixed. This may be even long-term, if additional manipulated variables are available. The mechanisms are relatively simple and consist in the reconfiguration of the model structure and the introduction of appropriate constraints into the optimization problem of the optimizer, thus not affecting the numerical effectiveness. Simulation results of the presented control system for a multivariable plant are provided, illustrating the efficiency of the proposed approach.
\end{abstract}

Keywords: fault-tolerant control, model predictive control, set-point optimization, nonlinear systems.

\section{Introduction}

On-line set-point optimization is one of the main issues in modern control systems, as economically optimal setpoints fluctuate with changes in disturbance inputs, in process parameters and external requirements. When all these changes are much slower (or abrupt but rare) than the dynamics of the feedback controlled process, then the standard multilayer control system structure, with steadystate economic optimization (usually using a comprehensive nonlinear process model) in a higher optimization layer, repeated much less frequently than the sampling periods of underlying feedback controllers, usually leads to satisfactory results. However, in practice it is often not the case, as the dynamical changes of disturbances can be of a similar variability as the dynamic processes in the controlled plant. Then the classical structure described above is not sufficient and the set-points should be optimized more frequently. The best solution would be to repeat the nonlinear steady-state model adaptation and economic steady-state optimization more frequently, even as frequently as the feedback controllers execute. However, this is usually not realistic due to the complexity of these tasks. If the underlying feedback control structure consists of two layers: a feedback control layer and a supervi- sory advanced (Model Predictive Control - MPC) control layer, then usually supervisory MPC controllers not only perform feedback control and constraint handling tasks, but are also supplemented with a simple steady-state optimization recalculating optimal set-points to counteract constraint violation and performance deterioration due to more frequent disturbance changes (Blevins et al., 2003; Kassmann et al., 2000; Qin and Badgwell, 2003; Tatjewski, 2007). A possible solution here is also a unified approach, where the optimization tasks of both the predictive controller and the set-point optimizer are integrated (Tvrzska de Gouvea and Odloak, 1998; Zanin et al., 2000; 2002; Ławryńczuk et al., 2007a). In the cases when the supervisory feedback control layer is not needed, one of the sound solutions can be to apply a supervisory predictive set-point optimizer, capable of adjusting the set-points in a way to control both optimality and constraint satisfaction (as basic feedback controllers are usually unconstrained, of a PID or an MPC type), as initially proposed in (Ławryńczuk et al., 2007b). The presentation of the optimizer and the possibilities of handling actuator faults in the resulting control structure is the subject of this paper. The goal of the optimizer is to generate possibly optimal set-points for the feedback controllers in such a way that also the constraints put on manipulated and output vari- 
ables are fulfilled. To achieve this goal, the optimizer uses not only models of the process (dynamic and static) but also models of the basic feedback controllers. The algorithm of the optimizer is formulated in such a way that only a numerically efficient, quadratic optimization problem must be solved at each sampling instant.

Model-based predictive algorithms are particularly susceptible to modifications capable of coping with actuator or sensor faults due to the possibility of appropriate modifications of the process model and the constraints (Marusak, 2007a-b; Marusak and Tatjewski, 2004). In particular, it is possible to incorporate mechanisms of fault tolerance to actuator faults into the discussed control structure with the predictive set-point optimizer. As it is now practically a standard that information about actuator faults can be made available for the supervisory control systems, see, e.g., (Qin and Badgwell, 2003), it is assumed in the paper that fault detection and isolation methods yielding such information are applied. In particular, it is assumed that actuator output measurements are available. In such a case the detection and isolation of the fault and the resulting control algorithm reconfiguration can be done practically during one sampling instant. The reader interested in process diagnostics may find information about this topic, e.g., in (Korbicz et al., 2004; Kościelny, 2001; Venkatasubramanian et al., 2003; Yen and Ho, 2003; Zhang, 2007) and in the references therein. Information concerning, particularly, actuator fault diagnosis can be found, e.g., in (Lunze and Supavatanakul, 2002; Lunze and Schröder, 2004; Blanke et al., 2006).

The mechanisms of fault tolerance proposed in the paper consist in adding equality constraints into the optimization problem solved at each iteration by the set-point optimizer, as soon as the failure is detected. These equality constraints do not change the properties of the problem they are added to, and the essential advantage of the predictive set-point optimizer discussed in the paper, i.e., its numerical efficiency, being a result of its formulation as a quadratic programming problem, is preserved. Moreover, it should be emphasized that the control system under consideration is nonlinear with set-point optimizer using, among others, a nonlinear steady-state process model (exploited during fault accommodation). Additionally, constraints on both the manipulated and output variables are taken into consideration by the optimizer. The reconfiguration solutions proposed so far utilized linear controllers that do not take into consideration the constraints existing in the system, see, e.g., (Lunze and Steffen, 2006; Richter et al., 2007, Staroswiecki et al., 2007) and references therein. The systems discussed in the paper, due to the character of diagnostic signals, are in fact hybrid systems. As the analysis of properties and, in particular, the stability of this kind of system is not the main topic of the paper, for details the reader is referred to (Bemporad et al., 2000a; Bemporad et al., 2000b; Biswas et al., 2005;
Mignone et al., 2000).

The investigation of properties of the fault-tolerant control systems proposed in the paper is conducted using an example of a control system of a nonlinear MIMO plant affected by actuator faults. The problem of theoretical analysis of the discussed control system, which utilizes successive linear approximation of the steady-state process model, is difficult and dependent on the properties of the control plant, as it is usually the case with nonlinear problems. Therefore, simulation, as an investigation method, is applied.

In the next section the predictive feedback controller will be first reminded, in particular, an unconstrained one. Section 3 contains a description of the predictive set-point optimizer. In Section 4 the mechanisms introducing tolerance to actuator faults into the control system under consideration are discussed. Section 5 contains a description of simulation experiments, performed in the control system of a MIMO nonlinear plant (an evaporator), indicating the efficiency of the proposed solutions. The paper is summarized in Section 6.

\section{Predictive control algorithms}

In the receding horizon predictive control algorithms manipulated variables are generated using the prediction of future behavior of the control plant, taking into account the constraints that exist in the control system. This makes it possible to create favorable mechanisms taking into consideration information about faults that occurred in the system, see, e.g., (Marusak, 2006; 2007a-b; Marusak and Tatjewski, 2004). Typically, the predictive control algorithms are formulated as minimization problems of a performance function subject to the constraints imposed on manipulated and output variables, see, e.g., (Camacho and Bordons, 1999; Maciejowski, 2002; Rossiter, 2003; Tatjewski, 2007):

$$
\min _{\Delta \boldsymbol{u}} \sum_{j=1}^{n_{y}} \sum_{i=1}^{p} \kappa_{j} \cdot\left(\bar{y}_{k}^{j}-y_{k+i \mid k}^{j}\right)^{2}+\sum_{j=1}^{n_{u}} \sum_{i=0}^{s-1} \lambda_{j} \cdot\left(\Delta u_{k+i \mid k}^{j}\right)^{2}
$$

subject to the constraints

$$
\begin{gathered}
\Delta u_{\min }^{j} \leq \Delta u_{k+i \mid k}^{j} \leq \Delta u_{\max }^{j}, \\
\quad i=0, \ldots, s-1, \quad j=1, \ldots, n_{u}, \\
u_{\min }^{j} \leq u_{k+i \mid k}^{j} \leq u_{\max }^{j}, \\
\quad i=0, \ldots, s-1, \quad j=1, \ldots, n_{u}, \\
y_{\min }^{j} \leq y_{k+i \mid k}^{j} \leq y_{\max }^{j}, \\
i=1, \ldots, p, \quad j=1, \ldots, n_{y},
\end{gathered}
$$

where $\bar{y}_{k}^{j}$ is a set-point value for the $j$-th output, $y_{k+i \mid k}^{j}$ is the $j$-th output value for the $(k+i)$-th sampling instant predicted at the $k$-th sampling instant using the control 
plant model (it depends on past and future values of manipulated variables), $u_{k+i \mid k}^{j}$ are future values and $\Delta u_{k+i \mid k}^{j}$ are future changes in the manipulated variables, $\kappa_{j} \geq 0$ and $\lambda_{j} \geq 0$ are weighting coefficients, $p$ and $s$ denote prediction and control horizons, respectively, and $n_{y}, n_{u}$ denote the number of outputs and inputs, respectively. Generally, the constraints on the output values (4) may cause emptiness of the admissible set of the problem (1)-(4). This is the reason why these constraints are usually treated as soft constraints. We omit the presentation of the practically important but standard modifications of the optimization problem (1)-(4) to treat the constraints (4) as soft ones, as the techniques are well known in the predictive control literature, see, e.g., (Camacho and Bordons, 1999; Maciejowski, 2002; Tatjewski, 2007).

The optimal vector of changes in the manipulated variables is obtained as a solution to the optimization problem (1)-(4). From this vector, the elements $\Delta u_{k \mid k}^{j}$, corresponding to the current sampling instant, are taken and applied in the control system. Then optimization is repeated at the next sampling instant.

It should be pointed out that the way the predicted output values $y_{k+i \mid k}^{j}$ are derived depends on the kind of the plant model exploited by the algorithm. If the linear model is used, then the problem (1)-(4) is a standard quadratic programming problem. Unfortunately, using the algorithm based on a linear plant model for the nonlinear plant may be insufficient if control in a wide range of setpoints is needed. On the other hand, if a nonlinear model is used, then the problem (1)-(4) is, in general, nonlinear and nonconvex instead of linear-quadratic. In such a case the computational burden needed to solve this problem can be prohibitive making practical implementation of the predictive algorithm more difficult and unreliable. A possible solution to these difficulties could be to use fuzzy predictive controllers based on a collection of linear models or controllers based on an on-line linearization approach, see, e.g., (Tatjewski, 2007) and a survey paper (Mayne et al., 2000), where also other approaches to the predictive control based on nonlinear models are given or referenced.

Let us introduce the following vectors:

$$
\begin{aligned}
\boldsymbol{y} & =\left[\boldsymbol{y}_{k}^{1}, \boldsymbol{y}_{k}^{2}, \ldots, \boldsymbol{y}_{k}^{n_{y}}\right]^{T}, \\
\boldsymbol{y}_{k}^{j} & =\left[y_{k+1 \mid k}^{j}, \ldots, y_{k+p \mid k}^{j}\right]^{T}, \\
\Delta \boldsymbol{u} & =\left[\Delta \boldsymbol{u}_{k}^{1}, \Delta \boldsymbol{u}_{k}^{2}, \ldots, \Delta \boldsymbol{u}_{k}^{n_{u}}\right]^{T}, \\
\Delta \boldsymbol{u}_{k}^{j} & =\left[\Delta u_{k \mid k}^{j}, \ldots, \Delta u_{k+s-1 \mid k}^{j}\right]^{T}, \\
\boldsymbol{u} & =\left[\boldsymbol{u}_{k}^{1}, \boldsymbol{u}_{k}^{2}, \ldots, \boldsymbol{u}_{k}^{n_{u}}\right]^{T}, \\
\boldsymbol{u}_{k}^{j} & =\left[u_{k \mid k}^{j}, \ldots, u_{k+s-1 \mid k}^{j}\right]^{T},
\end{aligned}
$$

and, by analogy, the vectors $\boldsymbol{u}_{\min }, \boldsymbol{u}_{\max }$ and $\Delta \boldsymbol{u}_{\min }$, $\Delta \boldsymbol{u}_{\max }$ of lower and upper bounds on the values and changes of the manipulated variables $\boldsymbol{u}$, and $\boldsymbol{y}_{\min }, \boldsymbol{y}_{\max }$ vectors of lower and upper bounds on the values of the output variables $\boldsymbol{y}$. Using this vector notation, the performance index (1) can be described by the following equation:

$$
J_{M P C}=(\overline{\bar{y}}-\boldsymbol{y}) \cdot \boldsymbol{\kappa} \cdot(\overline{\bar{y}}-\boldsymbol{y})+\Delta \boldsymbol{u}^{T} \cdot \boldsymbol{\lambda} \cdot \Delta \boldsymbol{u},
$$

where

$$
\overline{\bar{y}}=\left[\overline{\boldsymbol{y}}_{k}^{1}, \overline{\boldsymbol{y}}_{k}^{2}, \ldots, \overline{\boldsymbol{y}}_{k}^{n_{y}}\right]^{T}
$$

and every vector $\overline{\boldsymbol{y}}_{k}^{j}=\left[\bar{y}_{k}^{j}, \ldots, \bar{y}_{k}^{j}\right]^{T}$ is of length $p$, $\boldsymbol{\kappa}=\left[\boldsymbol{\kappa}_{1}, \ldots, \boldsymbol{\kappa}_{n_{y}}\right] \cdot \boldsymbol{I}$, every $\boldsymbol{\kappa}_{j}=\left[\kappa_{j}, \ldots, \boldsymbol{\kappa}_{j}\right]$ is of length $p, \boldsymbol{\lambda}=\left[\boldsymbol{\lambda}_{1}, \ldots, \boldsymbol{\lambda}_{n_{u}}\right] \cdot \boldsymbol{I}$, every $\boldsymbol{\lambda}_{j}=\left[\lambda_{j}, \ldots, \lambda_{j}\right]$ is of length $s$.

If the prediction is performed using a linear process model, then the superposition principle can be used and the vector of predicted output values $\boldsymbol{y}$ can be decomposed in the following way:

$$
\boldsymbol{y}=\widetilde{\boldsymbol{y}}\left(\boldsymbol{u}^{p}, \boldsymbol{y}^{p}\right)+\boldsymbol{A} \cdot \Delta \boldsymbol{u},
$$

where

$$
\widetilde{\boldsymbol{y}}\left(\boldsymbol{u}^{p}, \boldsymbol{y}^{p}\right)=\left[\widetilde{\boldsymbol{y}}_{k}^{1}, \widetilde{\boldsymbol{y}}_{k}^{2}, \ldots, \widetilde{\boldsymbol{y}}_{k}^{n_{y}}\right]^{T},
$$

$\widetilde{\boldsymbol{y}}_{k}^{j}=\left[\widetilde{y}_{k+1 \mid k}^{j}, \ldots, \widetilde{y}_{k+p \mid k}^{j}\right]^{T}$ is the free response of the control plant, $\boldsymbol{A}$ is a dynamic matrix composed of coefficients of the control plant step response:

$$
\begin{gathered}
\boldsymbol{A}=\left[\begin{array}{cccc}
\boldsymbol{A}_{11} & \boldsymbol{A}_{12} & \ldots & \boldsymbol{A}_{1 n_{u}} \\
\boldsymbol{A}_{21} & \boldsymbol{A}_{22} & \ldots & \boldsymbol{A}_{2 n_{u}} \\
\vdots & \vdots & \ddots & \vdots \\
\boldsymbol{A}_{n_{y} 1} & \boldsymbol{A}_{n_{y} 2} & \ldots & \boldsymbol{A}_{n_{y} n_{u}}
\end{array}\right], \\
\boldsymbol{A}_{m n}=\left[\begin{array}{ccccc}
a_{1}^{m, n} & 0 & \ldots & 0 & 0 \\
a_{2}^{m, n} & a_{1}^{m, n} & \ldots & 0 & 0 \\
\vdots & \vdots & \ddots & \vdots & \vdots \\
a_{p}^{m, n} & a_{p-1}^{m, n} & \ldots & a_{p-s+2}^{m, n} & a_{p-s+1}^{m, n}
\end{array}\right],
\end{gathered}
$$

$a_{i}^{m, n}\left(i=1, \ldots, p_{d}\right)$ are step response coefficients of the control plant describing the influence of the $n$-th input on the $m$-th output, $p_{d}$ is equal to the number of sampling instants after which the coefficients of the step responses can be assumed as settled $\left(p_{d} \geq p\right)$, see, e.g., (Tatjewski, 2007).

The free response $\widetilde{\boldsymbol{y}}\left(\boldsymbol{u}^{p}, \boldsymbol{y}^{p}\right)$ depends, in general, on vectors of values of manipulated and output variables from the past $\boldsymbol{u}^{p}, \boldsymbol{y}^{p}$, and its form depends on the kind of the process model that was used to obtain the prediction.

In the case of the DMC control algorithm, step responses are used as the model

$$
\hat{y}_{k}^{m}=\sum_{n=1}^{n_{u}} \sum_{j=1}^{p_{d}-1} a_{j}^{m, n} \cdot \Delta u_{k-j}^{n}+a_{p_{d}}^{m, n} \cdot u_{k-p_{d}}^{n},
$$


where $\hat{y}_{k}^{m}$ is the $m$-th output of the control plant model at the $k$-th sampling instant, $\Delta u_{k}^{n}$ is a change of the $n$-th manipulated variable at the $k$-th sampling instant, $u_{k-p_{d}}^{n}$ is the value of the $n$-th manipulated variable at the $(k-$ $p_{d}$ )-th sampling instant. The predicted values of output variables are described by

$$
y_{k+i \mid k}^{m}=\hat{y}_{k+i}^{m}+d_{k}^{m}
$$

where $d_{k}^{m}=y_{k}^{m}-\hat{y}_{k-1}^{m}$ is assumed the same at each sampling instant in the prediction horizon (a DMC-type model of unmeasured disturbances). Thus, the final formula is as follows:

$$
\begin{aligned}
y_{k+i \mid k}^{m}= & y_{k}^{m}+\sum_{n=1}^{n_{u}} \sum_{j=i+1}^{p_{d}-1} a_{j}^{m, n} \cdot \Delta u_{k-j+i}^{n} \\
& +a_{p_{d}}^{m, n} \cdot \sum_{n=1}^{n_{u}} \sum_{j=p_{d}}^{p_{d}+i-1} \Delta u_{k-j+i}^{n} \\
& -\sum_{n=1}^{n_{u}} \sum_{j=1}^{p_{d}-1} a_{j}^{m, n} \cdot \Delta u_{k-j}^{n} \\
& +\sum_{n=1}^{n_{u}} \sum_{j=1}^{i} a_{j}^{m, n} \cdot \Delta u_{k-j+i \mid k}^{n} .
\end{aligned}
$$

In (11) only the last component depends on future changes in the manipulated variable. The rest of the prediction is the element of the free response. Thus, rewriting the predictions in a vector form, one obtains (6).

The prediction was detailed for the step response model because it was used in the example. However, in the case of algorithms that are based on difference equations, the prediction also can be divided into the part dependent on past values of output and manipulated variables and the part dependent on the future changes of the manipulated variables, see, e.g., (Camacho and Bordons 1999; Maciejowski, 2002; Tatjewski, 2007). Thus, the prediction is still expressed in the form (6) but the free response is different (it depends not only on past values of manipulated variables but also on past values of output variables). Moreover, it can be shown that the part depending on decision variables is still described by the dynamic matrix, the same as in the DMC algorithm. In fact, it is the case for all predictive control algorithms regardless of the model they are based on provided that this model is linear. For details, see (Tatjewski, 2007).

The performance index (5) together with the prediction (6) can be written in the following form:

$$
\begin{aligned}
J_{M P C}= & (\boldsymbol{A} \cdot \Delta \boldsymbol{u}-\widetilde{\bar{y}})^{T} \cdot \boldsymbol{\kappa} \cdot(\boldsymbol{A} \cdot \Delta \boldsymbol{u}-\widetilde{\bar{y}}) \\
& +\Delta \boldsymbol{u}^{T} \cdot \boldsymbol{\lambda} \cdot \Delta \boldsymbol{u}
\end{aligned}
$$

where $\widetilde{\bar{y}}=\overline{\bar{y}}-\widetilde{\boldsymbol{y}}\left(\boldsymbol{u}^{p}, \boldsymbol{y}^{p}\right)$
If it is minimized without taking constraints into consideration, then the following analytical, unconstrained solution is obtained:

$$
\Delta \boldsymbol{u}=\left(\boldsymbol{A}^{T} \cdot \boldsymbol{\kappa} \cdot \boldsymbol{A}+\boldsymbol{\lambda}\right)^{-1} \cdot \boldsymbol{A}^{T} \cdot \boldsymbol{\kappa} \cdot \widetilde{\bar{y}}
$$

The above formula represents a linear feedback control law, generally with feedback from past process inputs and outputs (Camacho and Bordons, 1999; Maciejowski, 2002; Tatjewski, 2007). During the last years an alternative approach has emerged. It consists in designing an explicit piecewise-linear MPC control law, see, e.g., (Bemporad et al., 2002; Jones et al., 2007; Kerrigan and Maciejowski, 2004; Tondell et al., 2003). It can be a very efficient approach for relatively simple problems. However, in general, the number of controllers the algorithm switches between may happen to be significant, which is the main drawback of the approach.

\section{Predictive set-point optimizer}

In the standard multilayer structure consisting of a separate feedback control layer and a steady-state economic optimization layer, the set-point values for the feedback controllers are calculated in the economic optimization layer much less frequently than the feedback controllers execute (say, every hour as compared to every minute (Qin and Badgwell, 2003)). The following optimization problem is solved in the steady-state optimization layer:

$$
\min _{\overline{\boldsymbol{y}}, \overline{\boldsymbol{u}}} J_{E}(\overline{\boldsymbol{y}}, \overline{\boldsymbol{u}})
$$

subject to

$$
\begin{gathered}
\overline{\boldsymbol{u}}_{\min } \leq \overline{\boldsymbol{u}} \leq \overline{\boldsymbol{u}}_{\max }, \\
\overline{\boldsymbol{y}}_{\min } \leq \overline{\boldsymbol{y}} \leq \overline{\boldsymbol{y}}_{\max }, \\
\overline{\boldsymbol{y}}=F(\overline{\boldsymbol{u}}, \widetilde{\boldsymbol{w}}),
\end{gathered}
$$

where $F: \mathbb{R}^{n_{u}} \times \mathbb{R}^{n_{w}} \rightarrow \mathbb{R}^{n_{y}}, F \in C^{1}$ is a steadystate plant model, usually nonlinear, $n_{w}$ is the number of disturbances affecting the plant, $\overline{\boldsymbol{y}}$ is a vector of dimension $n_{y}$ of the set-point values, $\overline{\boldsymbol{u}}$ is a vector (of dimension $n_{u}$ ) of steady-state values of manipulated variables related to $\overline{\boldsymbol{y}}$ through the steady-state plant model, $\widetilde{\boldsymbol{w}}$ is a current disturbance estimate, $\overline{\boldsymbol{u}}_{\text {min }}, \overline{\boldsymbol{u}}_{\text {max }}$ are vectors of lower and upper bounds on manipulated variables $\overline{\boldsymbol{u}}$, and $\overline{\boldsymbol{y}}_{\min }, \overline{\boldsymbol{y}}_{\text {max }}$ are vectors of lower and upper bounds on the output values $\overline{\boldsymbol{y}}$.

The control structure discussed in this paper and shown in Fig. 1 operates in a different manner. The optimization problem is solved by the predictive set-point optimizer at the same sampling frequency as the feedback controllers execute. It can be formulated as a modification of the problem (14)-(17) of the steady-state economic optimization, as initially indicated in (Ławryńczuk et. al, 2007b). The modification consists in: adding to 


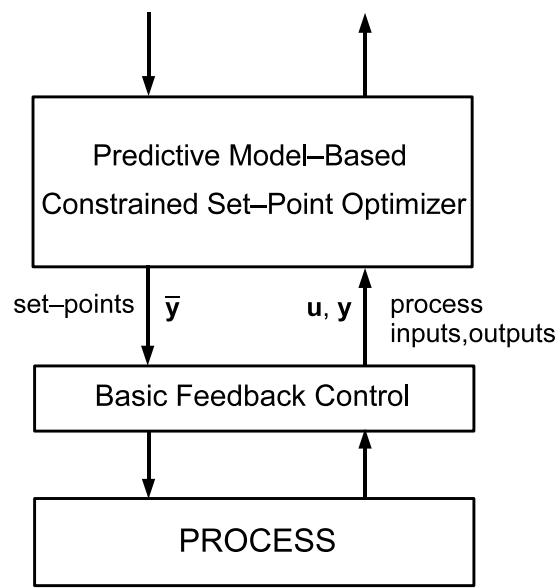

Fig. 1. Control system structure considered.

this problem the constraints (2)-(4) in the form used in the predictive control algorithm, using a linearized version of the steady-state process model (17) (linearization may be performed at each iteration or less frequently, if it is sufficient), and adding the dynamic models of the process and of the feedback controllers (or together, the dynamic model of the process with the controllers). The optimization problem solved by the optimizer has thus the following form:

$$
\min _{\overline{\boldsymbol{y}}, \overline{\boldsymbol{u}}} J_{E}(\overline{\boldsymbol{y}}, \overline{\boldsymbol{u}})
$$

subject to

$$
\begin{gathered}
\overline{\boldsymbol{u}}_{\min } \leq \overline{\boldsymbol{u}} \leq \overline{\boldsymbol{u}}_{\max }, \\
\overline{\boldsymbol{y}}_{\min } \leq \overline{\boldsymbol{y}} \leq \overline{\boldsymbol{y}}_{\max }, \\
\overline{\boldsymbol{y}}=F\left(\boldsymbol{u}_{k-1}, \widetilde{\boldsymbol{w}}_{k}\right)+\boldsymbol{H}_{k} \cdot\left(\overline{\boldsymbol{u}}-\boldsymbol{u}_{k-1}\right), \\
\Delta u_{\min }^{j} \leq \Delta u_{k+i \mid k}^{j} \leq \Delta u_{\max }^{j}, \\
i=0, \ldots, s-1, \quad j=1, \ldots, n_{u}, \\
u_{\min }^{j} \leq u_{k+i \mid k}^{j} \leq u_{\max }^{j}, \\
i=0, \ldots, s-1, \quad j=1, \ldots, n_{u}, \\
y_{\min }^{j} \leq y_{k+i \mid k}^{j} \leq y_{\max }^{j}, \\
i=1, \ldots, p, \quad j=1, \ldots, n_{y}, \\
\boldsymbol{y}=\widetilde{\boldsymbol{y}}\left(\boldsymbol{u}^{p}, \boldsymbol{y}^{p}\right)+\boldsymbol{A} \cdot \Delta \boldsymbol{u}, \\
\boldsymbol{u}=R\left(\overline{\boldsymbol{y}}, \boldsymbol{y}^{p}, \boldsymbol{u}^{p}\right),
\end{gathered}
$$

where (21) is a linearization of the steady-state plant model, $\boldsymbol{u}_{k-1}$ is a vector of manipulated variable values applied to the plant at the last sampling instant, $\boldsymbol{H}_{k}$ is the $n_{y} \times n_{u}$ matrix that contains the partial derivatives of the function $\overline{\boldsymbol{y}}=F(\overline{\boldsymbol{u}}, \widetilde{\boldsymbol{w}})$, see, e.g., (Ławryńczuk et al., 2007b) for details.

It is a key feature of the optimizer that it uses not only the steady-state plant model (21) but also a dynamic model of the controlled plant, as the predicted output variable values $y_{k+i \mid k}^{j}\left(i=1, \ldots, p, j=1, \ldots, n_{y}\right)$ are calculated iteratively on the prediction horizon using a feedback controlled plant model. This can be implemented as a separate dynamic model of the plant (25) and the model of the feedback controllers (26). These are typically PID controllers or simple unconstrained MPC controllers (13). Thus their models are linear functions of set-points $\bar{y}$ and past values of the output variables $y_{k-i}^{j}$ and the manipulated variables $u_{k-i}^{j}$. Therefore, in the case of the PID controllers, (26) becomes

$$
\boldsymbol{u}_{k}=\boldsymbol{u}_{k-1}+\boldsymbol{r}_{0} \cdot \boldsymbol{e}_{k}+\boldsymbol{r}_{1} \cdot \boldsymbol{e}_{k-1}+\boldsymbol{r}_{2} \cdot \boldsymbol{e}_{k-2},
$$

where $\boldsymbol{r}_{0}, \boldsymbol{r}_{1}, \boldsymbol{r}_{2}$ are vectors containing parameters of the controllers, $\boldsymbol{e}_{k-i}=\overline{\boldsymbol{y}}_{k-i}-\boldsymbol{y}_{k-i}$ are vectors containing values of control errors at the $(k-i)$-th sampling instants. In the case of the unconstrained MPC controllers, (26) becomes

$$
\boldsymbol{u}_{k}=\boldsymbol{u}_{k-1}+\left(\boldsymbol{A}^{T} \cdot \boldsymbol{\kappa} \cdot \boldsymbol{A}+\boldsymbol{\lambda}\right)^{-1} \cdot \boldsymbol{A}^{T} \cdot \boldsymbol{\kappa} \cdot \widetilde{\overline{\boldsymbol{y}}}
$$

A big advantage of the set-point optimizer is that it can be relatively easily designed for existing basic feedback control systems, extending their capabilities. Thanks to the inclusion of the controlled plant model and the constraints (22)-(24) in the optimization problem (18)-(26), the constraints put on the manipulated and output variables on the prediction horizon are taken into consideration during the set-point generation.

In the example discussed later, a DMC analytical controller (a special case of (13)) will be used as the feedback controller. It is also possible to use one combined model of the basic feedback controllers together with the plant. In the example, the PI controller was treated in this way.

\section{Actuator fault tolerance}

4.1. Modification of dynamic models. After detecting and isolating a fault, both the set-point optimizer and the basic feedback controllers should be appropriately modified. The first modification is based on the introduction of the following equality constraints into the control problem solved at each iteration by the optimizer (Marusak and Tatjewski, 2004):

$$
\Delta u_{k+i \mid k}^{f}=0, \quad i=0, \ldots, s-1,
$$

where $f$ is the number of the manipulated variable affected by the fault (blockade). It should be emphasized that the constraints (27) are imposed on all manipulated variable changes from the whole control horizon. The introduction of these constraints means in practice a modification of the dynamic control plant model used by the optimizer. 
The second modification is first introduced to the model of basic feedback controllers (26). If several SISO PID controllers are used, then the model of the appropriate PID controller should be removed from the set of equality constraints (26a) and its output replaced by the output value of the blocked actuator. In the case of analytical predictive controllers (26b), they should be reconfigured as described in (Marusak, 2007a) and their model, used by the set-point optimizer, should be updated. The reconfiguration consists in the modification of the dynamic matrix (7). The modification is relatively simple and consists in eliminating, from the dynamic matrix (7), the columns describing the dependence of output variables on the manipulated variable affected by the failure:

$$
\begin{aligned}
\boldsymbol{A}= & {\left[\begin{array}{cccc}
\boldsymbol{A}_{11} & \ldots & \boldsymbol{A}_{1(f-1)} & \boldsymbol{A}_{1(f+1)} \\
\boldsymbol{A}_{21} & \ldots & \boldsymbol{A}_{2(f-1)} & \boldsymbol{A}_{2(f+1)} \\
\vdots & \ddots & \vdots & \vdots \\
\boldsymbol{A}_{n_{y} 1} & \ldots & \boldsymbol{A}_{n_{y}(f-1)} & \boldsymbol{A}_{n_{y}(f+1)} \\
& \ldots \boldsymbol{A}_{1\left(n_{u}-1\right)} & \boldsymbol{A}_{1 n_{u}} \\
& \ldots & \boldsymbol{A}_{2\left(n_{u}-1\right)} & \boldsymbol{A}_{2 n_{u}} \\
& \ddots & \vdots & \vdots \\
& \ldots \boldsymbol{A}_{n_{y}\left(n_{u}-1\right)} & \boldsymbol{A}_{n_{y} n_{u}}
\end{array}\right] }
\end{aligned}
$$

where $f$ is the number of the manipulated variable affected by the actuator blockade.

The vector of future increments in the manipulated variables will also change:

$$
\Delta \boldsymbol{u}=\left[\Delta \boldsymbol{u}_{k}^{1}, \ldots, \Delta \boldsymbol{u}_{k}^{f-1}, \Delta \boldsymbol{u}_{k}^{f+1}, \ldots, \Delta \boldsymbol{u}_{k}^{n_{u}}\right]^{T},
$$

as well as the weighting matrix $\lambda$ :

$$
\boldsymbol{\lambda}=\left[\boldsymbol{\lambda}_{1}, \ldots, \boldsymbol{\lambda}_{f-1}, \boldsymbol{\lambda}_{f+1}, \ldots, \boldsymbol{\lambda}_{n_{u}}\right] \cdot \boldsymbol{I} .
$$

Thus, the performance index (12) and the control law (13) change. The updated control law should be also included in the formulation of the optimization problem solved by the set-point optimizer.

4.2. Modification of the steady-state model. The modification of a steady-state process model is based on the introduction of the following equality constraint:

$$
\bar{u}^{f}=u_{b l}^{f},
$$

where $f$ is the number of the manipulated variable affected by the fault, $u_{b l}^{f}$ is the output value of the actuator. The introduction of the constraint (31) into the integrated optimization problem (18)-(26) can substantially improve the quality of control, as it will be illustrated in an example. The reason is that this means an update of the steady-state process model $\overline{\boldsymbol{y}}=F(\overline{\boldsymbol{u}}, \widetilde{\boldsymbol{w}})$ used to calculate an economically optimal operating point. After such a change the controller possesses information that it has a limited possibility of achieving some set-point values $\bar{y}$ because of the actuator blockade. Thus, the optimizer can change the set-points appropriately till the failure is fixed.

4.3. Case with constrained outputs. In the case of constraints imposed on the values of the output variables it can be useful to introduce a safety zone near the constraints after the failure detection and isolation. In such a case one should modify the constraints (20) in the following way:

$$
\overline{\boldsymbol{y}}_{\min }+\overline{\boldsymbol{r}}_{\min } \leq \overline{\boldsymbol{y}} \leq \overline{\boldsymbol{y}}_{\max }-\overline{\boldsymbol{r}}_{\max }
$$

where $\overline{\boldsymbol{r}}_{\min }, \overline{\boldsymbol{r}}_{\max }$ are vectors shifting the output constraints; each component of such a vector is nonnegative $\left(\bar{r}_{\min }^{j} \geq 0, \bar{r}_{\max }^{j} \geq 0, j=1, \ldots, n_{y}\right)$. The introduction of nonzero elements in vectors $\overline{\boldsymbol{r}}_{\text {min }}, \overline{\boldsymbol{r}}_{\max }$ aims at taking into account the changes in the output signal that may increase after the fault occurrence (Marusak, 2006). The introduction of safety zones, as presented, results in fact in shifting the set-point values, which is a common practice in industry.

4.4. Application of an additional manipulated variable. If in the control system affected by the actuator blockade a new process input can be used as an additional manipulated variable, it can dramatically improve the obtained control performance, see, e.g., (Marusak, 2007a; Marusak and Tatjewski, 2004). In the case of analytical predictive controllers, such a change consists in the modification of the dynamic matrix. This time, columns describing the dependence of the output variables on the supplementary manipulated variable are added to the dynamic matrix (Marusak, 2007a):

$$
\boldsymbol{A}=\left[\begin{array}{cccc}
\boldsymbol{A}_{11} & \ldots & \boldsymbol{A}_{1 n_{u}} & \boldsymbol{A}_{1\left(n_{u}+1\right)} \\
\boldsymbol{A}_{21} & \ldots & \boldsymbol{A}_{2 n_{u}} & \boldsymbol{A}_{2\left(n_{u}+1\right)} \\
\vdots & \ddots & \vdots & \vdots \\
\boldsymbol{A}_{n_{y} 1} & \ldots & \boldsymbol{A}_{n_{y} n_{u}} & \boldsymbol{A}_{n_{y}\left(n_{u}+1\right)}
\end{array}\right] .
$$

Moreover, the vector of future increments in the manipulated variables changes to

$$
\Delta \boldsymbol{u}=\left[\Delta \boldsymbol{u}_{k}^{1}, \ldots, \Delta \boldsymbol{u}_{k}^{n_{u}}, \Delta \boldsymbol{u}_{k}^{n_{u}+1}\right]^{T}
$$

and the weighting matrix $\boldsymbol{\lambda}$ is modified as

$$
\boldsymbol{\lambda}=\left[\boldsymbol{\lambda}_{1}, \ldots, \boldsymbol{\lambda}_{n_{u}}, \boldsymbol{\lambda}_{n_{u}+1}\right] \cdot \boldsymbol{I} .
$$

As in the previous case, the control law (13) must be recalculated and the model of basic feedback controllers (26) updated. 
Remark 1. As is demonstrated in this section, the modifications that should be introduced into the control system in response to the actuator fault are just slightly complicated. This is because it is easy to add constraints to the optimization problem solved on-line at each algorithm iteration, anyway. If it is needed, also the process model may be changed with relative ease. Thus, the proposed fault accommodation mechanisms demand little effort (in fact, the optimization problem may become simpler when some decision variables are eliminated) and are very efficient, as is demonstrated in the next section, provided the diagnostic information is correct.

\section{Simulation experiments}

5.1. Control plant. The control plant under investigation is an evaporator, described thoroughly in (Newell and Lee, 1989), with one of the output variables controlled by means of a PI level controller. The diagram of the control plant is shown in Fig. 2.

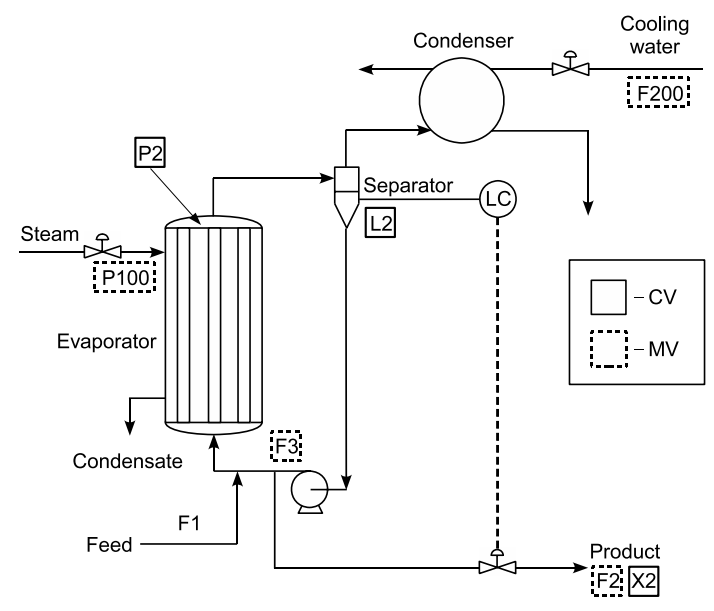

Fig. 2. Diagram of the control plant.

The output variables of the plant are: $L 2-$ level of the liquid in the separator (stabilized near $\bar{L} 2=1 \mathrm{~m}$ by a PI controller with parameters $K_{p}=5.6$ and $T_{i}=8.84 \mathrm{~min}$ ), $X 2$ - product composition, $P 2-$ pressure in the evaporator. Manipulated variables are: $F 2$-product flow (the variable used to stabilize $L 2$ level), $P 100$ - steam pressure, F200 - cooling water flow, $F 3$ - circulating flow (used in some experiments as a manipulated variable). It was also assumed that the feed flow rate $F 1$ is a measured disturbance. The notation is assumed the same as that in (Newell and Lee, 1989), where equations describing the control plant are presented. It is also assumed that the manipulated variables are constrained:

$$
\begin{aligned}
& P 100_{\text {min }} \leq P 100 \leq P 100_{\text {max }}, \\
& F 200_{\text {min }} \leq F 200 \leq F 200_{\text {max }},
\end{aligned}
$$

where $P 100_{\min }=0 \mathrm{kPa}, P 100_{\max }=400 \mathrm{kPa}$, $F 200_{\min }=0 \mathrm{~kg} / \mathrm{min}, F 200_{\max }=400 \mathrm{~kg} / \mathrm{min}$ (Newell and Lee, 1989).

5.2. Control system structure. For the control plant, an analytical, unconstrained DMC predictive controller and a set-point optimizer were designed. The manipulated variables of the controller are: steam pressure $P 100$, cooling water flow F200 and in some experiments circulating flow $F 3$, and the controlled variables are: product composition $X 2$ and pressure in the evaporator $P 2$. As a dynamic control plant model, the model composed of step responses obtained near the operating point $X 2=25 \%$, $P 2=42 \mathrm{kPa}$ was used. The following values of the controller parameters were assumed: $\kappa_{X 2}=\kappa_{P 2}=1$, $\lambda_{P 100}=\lambda_{F 200}=0.1, \lambda_{F 3}=5$, prediction horizon $p=100$, control horizon $s=10$.

It was also assumed that the product composition is constrained (a requirement on the quality of the product), with $X 2_{\min }=25 \%$. The following economic performance index was used:

$$
J_{E}=c_{1} \cdot \bar{P} 100-c_{2} \cdot \bar{F} 2,
$$

where $c_{1}=0.01$ and $c_{2}=1$ are the prices of the energy put into the process and of the product, respectively. The constraint imposed on the set-point $\bar{X} 2$ was as follows:

$$
\bar{X} 2_{\min }+\bar{r}_{\min }^{X 2} \leq \bar{X} 2,
$$

where $\bar{X} 2_{\min }=X 2_{\min }, \bar{r}_{\min }^{X 2}=1 \%$ is the value shifting the constraint, after the detection of a failure, in order to take into account changes in the output signal caused by the controller action.

5.3. Experiments. First, the experiments with control systems not affected by any failure were made, as the reference case for the remaining experiments. In Fig. 3, the case of the $X 2$ composition set-point constraint change $\bar{r}_{\min }^{X 2}=1 \%$ at the initial point of the simulation is depicted. Moreover, the disturbance $F 1$ (feed flow rate) changed from $F 1_{0}=10 \mathrm{~kg} / \mathrm{min}$ to $F 1_{0}=9.7 \mathrm{~kg} / \mathrm{min}$ at $t=100 \mathrm{~min}$, which resulted in a modified $P 2$ set-point trajectory calculated by the optimizer, as can be seen in Fig. 3.

In the second case, it was assumed that the disturbance signal $F 1$ was changing according to

$$
F 1(t)=F 1_{0}+F 1_{a} \cdot \sin \left(\frac{2 \pi t}{T_{o}}\right),
$$

where $F 1_{a}=0.35 \mathrm{~kg} / \mathrm{min}, T_{o}=250 \mathrm{~min}$. The obtained responses are shown in Fig. 4.

During the next experiment it was assumed that an actuator failure occurred at the beginning. After fault detection and isolation the safety zone is introduced into the 

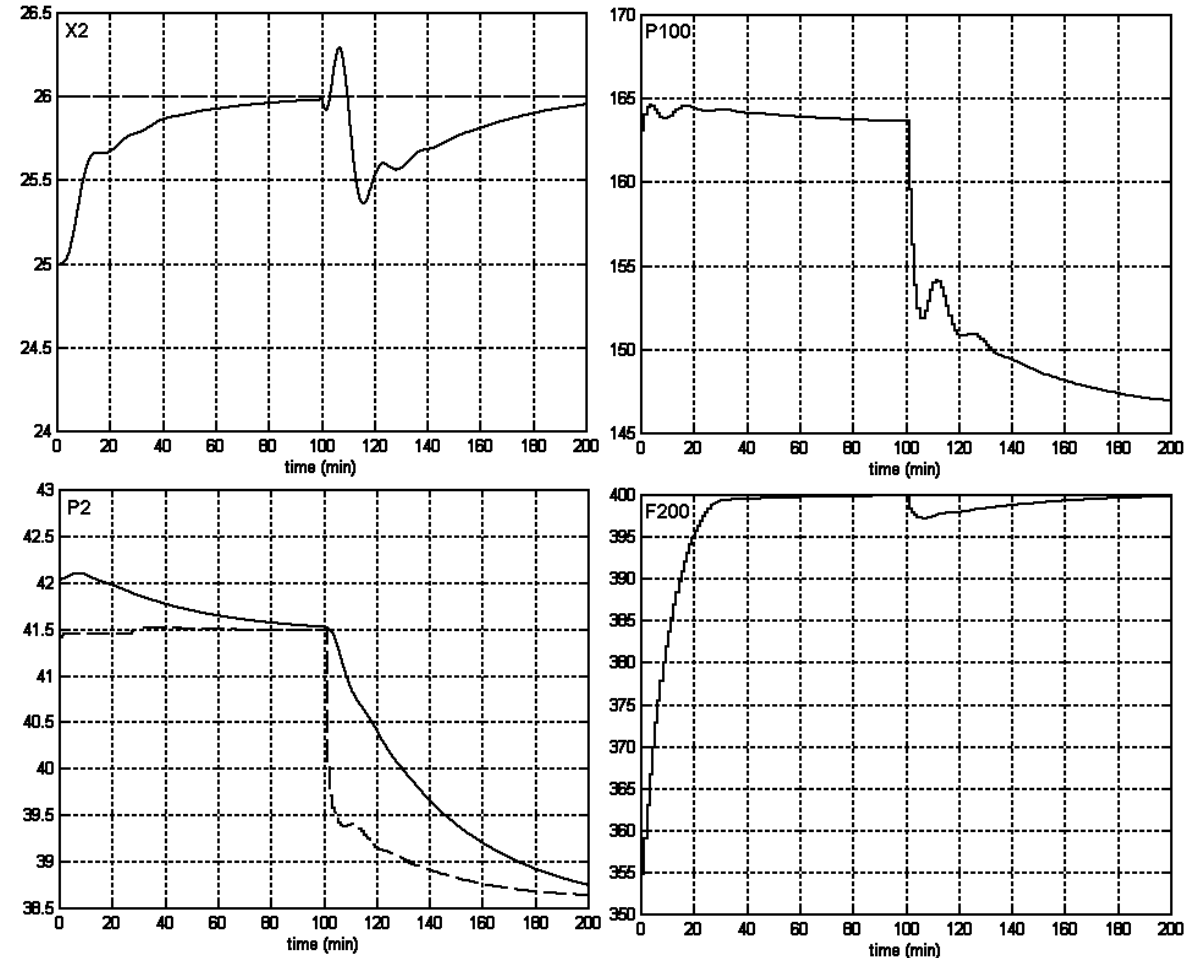

Fig. 3. Responses of the control system with a predictive set-point optimizer to a $X 2$ set-point constraint change and a step change in the disturbance $F 1$ from $F 1_{0}=10 \mathrm{~kg} / \mathrm{min}$ to $F 1_{0}=9.7 \mathrm{~kg} / \mathrm{min}$; left - outputs: $X 2$ and $P 2$ (dashed lines represent set-points), right - manipulated variables: $P 100$ and F200.
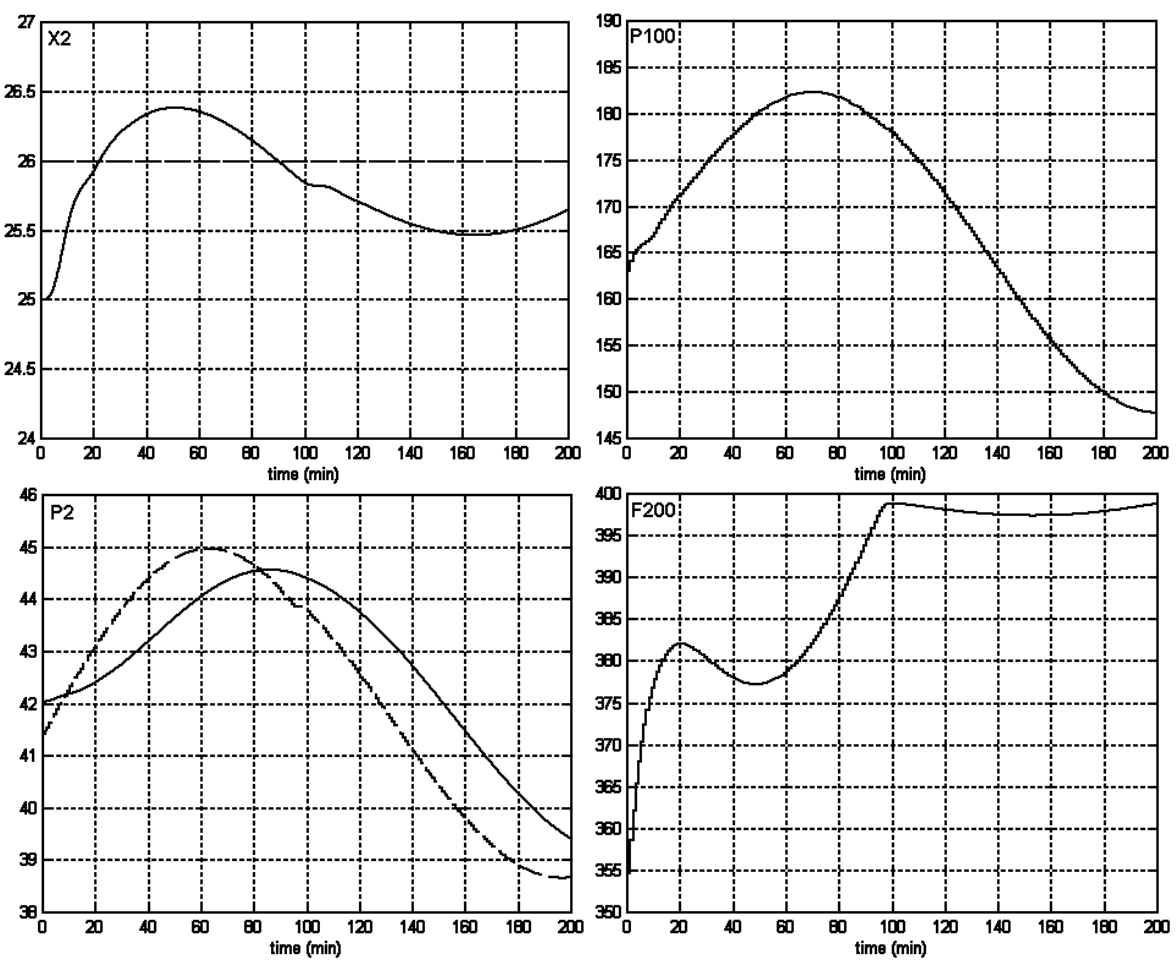

Fig. 4. Responses of the control system with a predictive set-point optimizer to a $X 2$ set-point constraint change and changes in the disturbance variable $F 1$; left - outputs: $X 2$ and $P 2$ (dashed lines represent set-points), right - manipulated variables: $P 100$ and $F 200$. 
constraint put on the set-point according to (39), in order to minimize the risk of its violation. In the 100-th minute of the experiment, the disturbance $F 1$ (feed flow rate) changed from $F 1_{0}=10 \mathrm{~kg} / \mathrm{min}$ to $F 1_{0}=9.7 \mathrm{~kg} / \mathrm{min}$. The results obtained in the case of the actuator blockage of the manipulated variable $F 200$ are presented in Fig. 5.

Before any mechanisms of fault accommodation were used, the output variables were far from their setpoint values and the $X 2$ composition constraint was violated between the 110-th and 150-th minutes (responses marked with dotted lines in Fig. 5). Unfortunately, only a slightly better result was obtained after the modification of the analytical controller and dynamic models in the setpoint optimizer (dashed lines in Fig. 5). Crucial for the control system performance was the modification of the steady-state process model by the inclusion of the constraint (31) into the optimization problem of the set-point optimizer. The response of the product composition $X 2$ achieves then the desired set-point and it is only near the 110-th minute that the constraint on $X 2$ is active for a short period of time (solid lines in Fig. 5).

In the next experiment it was assumed that a failure of the F200 manipulated variable actuator occurred at the beginning and that the disturbance signal $F 1$ was changing according to (40). The obtained results are presented in Fig. 6. This time, if none of the mechanisms of fault accommodation were used, the $X 2$ composition violated the constraint from the 120-th minute (dotted lines in Fig. 6). The economic optimization index calculated as a sum of temporary values of (38) was equal to 1003.8. (This means that losses were generated.) The modification of the analytical controller and dynamic models in the setpoint optimizer brought, unfortunately, a small improvement in control system operation (dashed lines in Fig. 6). Now, the performance index is equal to 967.1 (losses are a little bit smaller than in the case when nothing was done after the blockade). After the modification of the steadystate process model (the inclusion of the constraint (31) in the set-point optimizer), the response of the composition $X 2$ fulfills the assumed purity criterion (solid lines in Fig. 6) and the production yields profits - the performance index is equal to -419.3 .

5.3.1. Application of an additional manipulated variable. During the next experiment it was assumed that a failure of the $P 100$ manipulated variable actuator occurred at the beginning. Moreover, the disturbance $F 1$ changed at the 200-th minute, from $F 1_{0}=10 \mathrm{~kg} / \mathrm{min}$ to $F 1_{0}=10.5 \mathrm{~kg} / \mathrm{min}$. When the actuator blockade was not taken into consideration at all (dotted lines in Fig. 7), the composition $X 2$ settled very slowly and was far from the set-point value. After the change in the disturbance $F 1$, the obtained response is unacceptable-the product composition $X 2$ constraint is violated all the time starting from about the 205-th minute and the violation is huge.

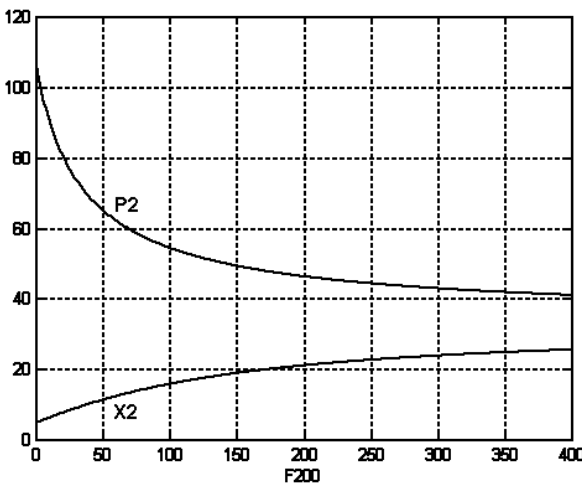

Fig. 8. Steady-state characteristics $X 2(F 200)$ and $P 2(F 200)$ of the control plant with a blockade of the actuator for the manipulated variable $P 100 ; F 1_{0}=10 \mathrm{~kg} / \mathrm{min}$.

The reconfiguration of the dynamic models in the setpoint optimizer changed the situation only a little (dashed lines in Fig. 7). After the change of the disturbance, the quality constraint is still violated (but the violation is not so big now).

An experiment was also made with a change in the steady-state control plant model. However, numerical problems with solving the optimization task of the setpoint optimizer occurred. The numerical procedure returned the message that there is no admissible set. After checking the steady-state characteristics of the plant it was found out that it was impossible to achieve composition $X 2=26 \%$ for the existing constraints (Fig. 8). This problem deepens with an increase in the value of the disturbance variable $F 1$. In such a case one should either reformulate the control goals or, if it is possible, use an additional manipulated variable. In the example considered, there is an input variable that can be used as the next manipulated variable; it is the circulating flow F3 (Newell and Lee, 1989).

After the application of the flow $F 3$ as the additional manipulated variable, much better control performance was obtained. This time, after the blockade of the $P 100$, it is possible to stabilize the product composition $X 2$ on the set-point value $26 \%$ (solid lines in Fig. 7), which was impossible before. Even after the change in the disturbance variable $F 1$, the product quality constraint is violated only during a limited period of time and then the composition $X 2$ settles on the set-point value $26 \%$.

\section{Conclusions}

A control structure with a predictive set-point optimizer supervising the plant with unconstrained feedback controllers was considered. The mechanisms of actuator fault toleration introduced into the set-point optimizer were proposed. They are easy to apply and efficient, making the task of optimization not more difficult than in 

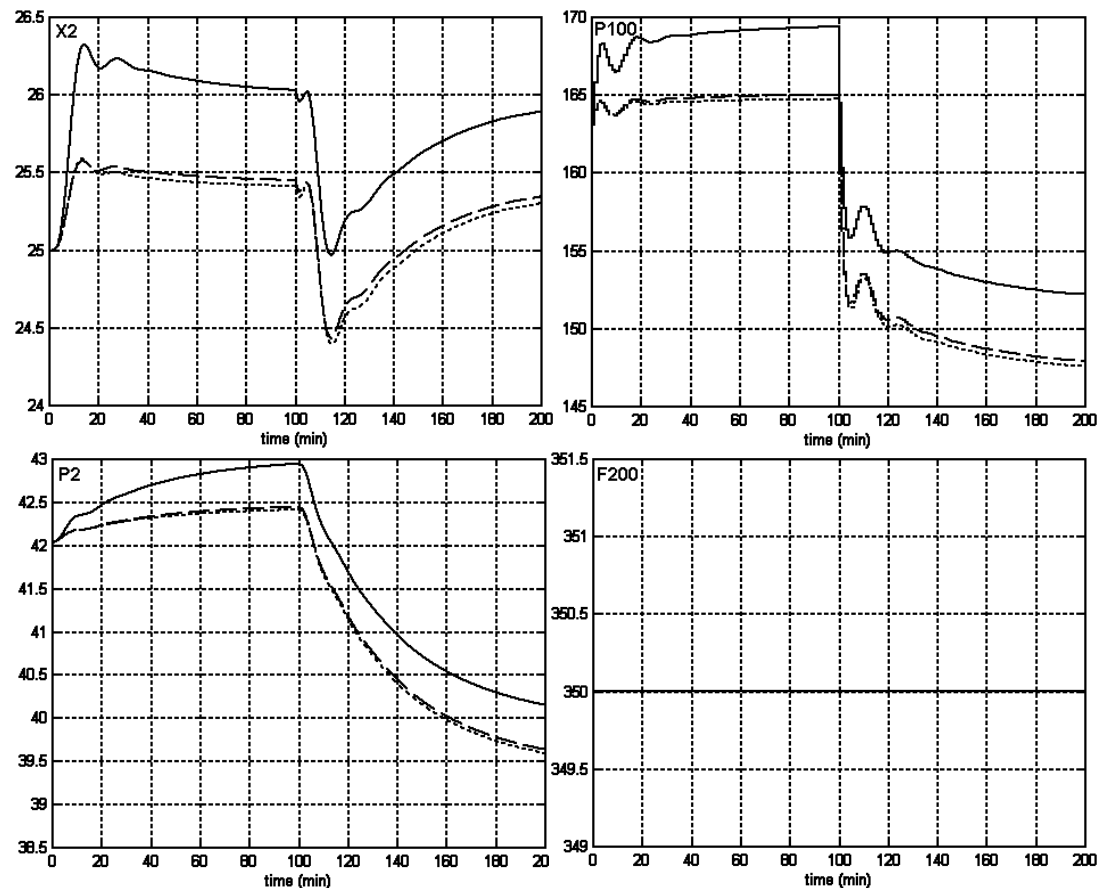

Fig. 5. Responses of the control system with a predictive set-point optimizer; a blockade of the actuator F200: not taken into consideration - dotted lines, taken into consideration by: adding only constraints (27) - dashed lines, adding also constraint (31) solid lines; left - outputs: $X 2$ and $P 2$ (dashed lines - set-points), right - manipulated variables: $P 100$ and F200.
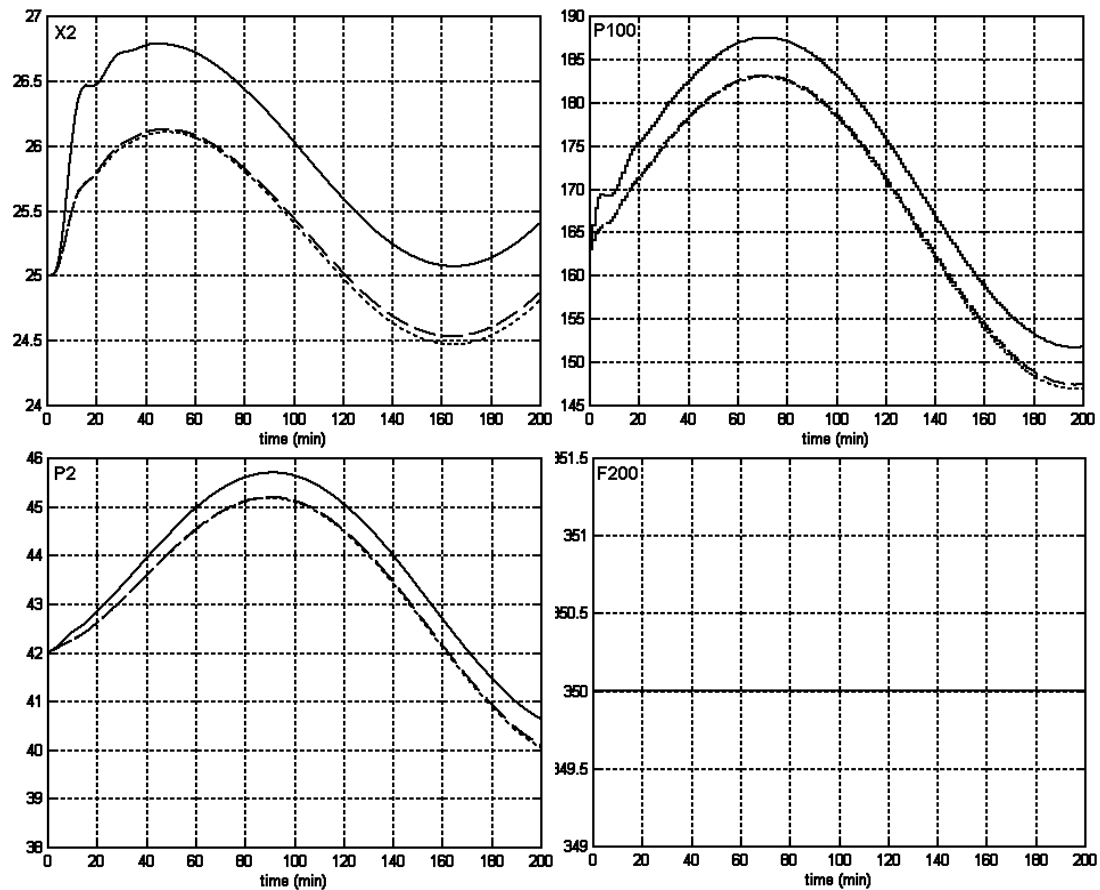

Fig. 6. Responses of the control system with a predictive set-point optimizer to changes in the disturbance $F 1$; a blockade of the actuator F200: not taken into consideration - dotted lines, taken into consideration by: adding only constraints (27) - dashed lines, adding also constraint (31) - solid lines; left - outputs: $X 2$ and $P 2$ (dashed lines - set-points), right - manipulated variables: $P 100$ and $F 200$. 

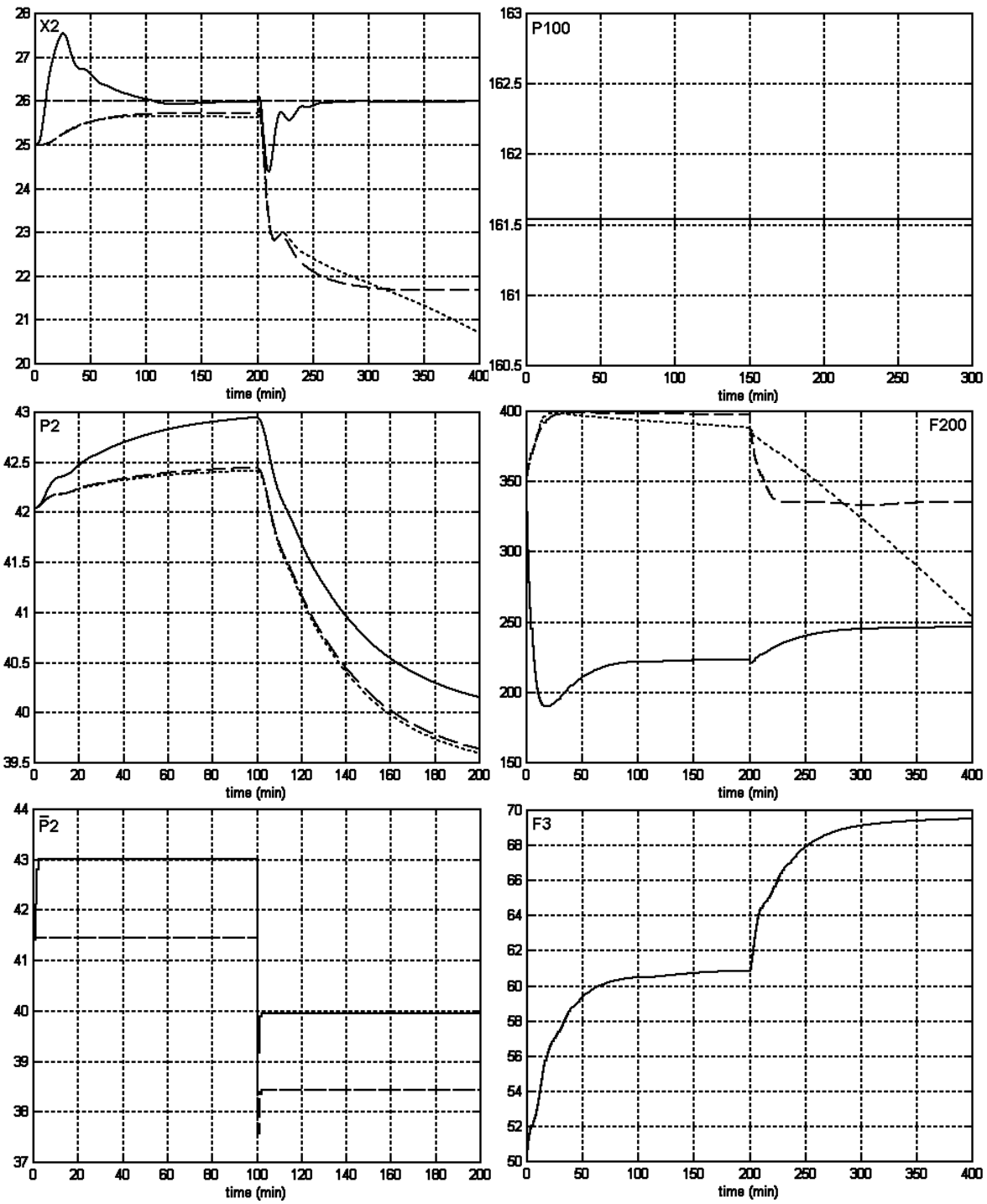

Fig. 7. Responses of the control system with a predictive set-point optimizer; a blockade of the actuator P100: not taken into consideration - dotted lines, taken into consideration by: adding only constraints (27) - dashed lines, taken into consideration and with additional manipulated variable - solid lines; left - outputs: $X 2, P 2$ and $P 2$ set-point, right - manipulated variables: $P 100$, $F 200$ and F3.

the case without faults. The optimization problem of the predictive set-point optimizer remains an easy-to-solve quadratic programming problem.

The proposed mechanisms were successfully used in the constrained control system of a nonlinear MIMO plant (an evaporator). An improvement in the control system operation was achieved despite the relative simplicity of the mechanisms applied. (They consist in adding certain constraints to the optimization problem solved at each iteration by the set-point optimizer.)

In the set-point optimizer both the linear dynamic model of the controlled plant and the on-line linearized steady-state process model are used. The use of the latter is essential for the effectiveness of the actuator fault ac- commodation mechanisms discussed in the paper. They rely on information about the actuator fault delivered as a diagnostic signal, usually from the actuator's selfdiagnostic system.

It is possible to improve the control system operation in the faulty situation if an additional manipulated variable can be used. In favorable conditions, it can even help to continue control system operation with the performance close to that before the occurrence of the fault.

\section{Acknowledgment}

This work was supported by the Polish national budget funds for science. 


\section{References}

Bemporad A., Borrelli F. and Morari M. (2000a). Optimal controllers for hybrid systems: Stability and piecewise linear explicit form, Proceedings of the 39-th IEEE Conference on Decision and Control, Sydney, Australia, pp. 18101815.

Bemporad A., Ferrari-Trecate G. and Morari M. (2000b). Observability and controllability of piecewise affine and hybrid systems, IEEE Transactions on Automatic Control 45(10): 1864-1876.

Bemporad A., Morari M., Dua V. and Pistikopoulos E. (2002). The explicit linear-quadratic regulator for constrained systems, Automatica 38(1): 3-20.

Biswas P., Grieder P., Löfberg J. and Morari M. (2005). A survey on stability analysis of discrete-time piecewise affine systems, Proceedings of the IFAC World Congress, Prague, Czech Republic, CD-ROM, paper no. Th-E12-TO/1.

Blanke M., Kinnaert M., Lunze J. and Staroswiecki M. (2006). Diagnosis and Fault-Tolerant Control, Springer-Verlag, Berlin.

Blevins T., McMillan G., Wojsznis W. and Brown M. (2003). Advanced Control Unleashed, ISA - The Instrumentation, Systems, and Automation Society, Research Triangle Park.

Camacho E. and Bordons C. (1999). Model Predictive Control, Springer-Verlag, London.

Jones C., Kerrigan E. and Maciejowski J. (2007). Lexicographic perturbation for multiparametric linear programming with applications to control, Automatica 43(10): 1808-1816.

Kassmann D., Badgwell T. and Hawkins R. (2000). Robust steady-state target calculation for model predictive control, AIChE Journal 46(5): 1007-1024.

Kerrigan, E. and Maciejowski J. (2004). Feedback min-max model predictive control using a single linear program: Robust stability and the explicit solution, International Journal of Robust and Nonlinear Control 14(4): 395-413.

Korbicz J., Kościelny J., Kowalczuk Z. and Cholewa W. (2004). Fault Diagnosis: Models, Artificial Intelligence, Applications, Springer-Verlag, Berlin.

Kościelny J. (2001). Diagnosis of Automated Industrial Processes, Academic Publishing House EXIT, Warsaw (in Polish).

Ławryńczuk M., Marusak P. and Tatjewski P. (2007a). Multilayer and integrated structures for predictive control and economic optimisation, Proceedings of the 11-th IFAC/IFORS/IMACS/IFIP Symposium on Large Scale Systems: Theory and Applications, Gdańsk, Poland, CDROM, paper no. 60.

Ławryńczuk M., Marusak P. and Tatjewski P. (2007b). Setpoint optimisation and predictive constrained control for fast feedback controlled processes, Proceedings of the 13-th IEEE/IFAC International Conference on Methods and Models in Automation and Robotics MMAR 2007, Szczecin, Poland, pp. 357-362.
Lunze J. and Schröder J. (2004). Sensor and actuator fault diagnosis of systems with discrete inputs and outputs, IEEE Transactions on Systems, Man, and Cybernetics, Part B: Cybernetics 34(2): 1096-1107.

Lunze J. and Steffen T. (2006). Control reconfiguration after actuator failures using disturbance decoupling methods, IEEE Transactions on Automatic Control 51(10): 1590 1601.

Lunze J. and Supavatanakul P. (2002). Timed discrete-event method for diagnosis of industrial actuators, Proceedings of the IEEE International Conference on Industrial Technology, Bangkok, Thailand, pp. 1354-1359.

Maciejowski J. (2002). Predictive Control with Constraints, Prentice Hall, Harlow.

Marusak P. (2006). Predictive control algorithms in constrained control systems tolerating sensor faults, Proceedings of the 12-th IEEE International Conference on Methods and Models in Automation and Robotics MMAR 2006, Międzyzdroje, Poland, pp. 797-804.

Marusak P. (2007a). Actuator fault toleration in control systems with analytical predictive controllers and output constraints, Proceedings of the 13-th IEEE/IFAC International Conference on Methods and Models in Automation and Robotics MMAR 2007, Szczecin, Poland, pp. 825-832.

Marusak P. (2007b). Predictive controllers integrated with economic optimization tolerating actuator faults: Application to a nonlinear plant, in J. Korbicz, K. Patan and M. Kowal (Eds), Fault Diagnosis and Fault Tolerant Control, Academic Publishing House EXIT, Warsaw, pp. 173-185.

Marusak P. and Tatjewski P. (2004). Predictive control algorithms in systems tolerating actuator faults, Proceedings of the 10-th IEEE International Conference on Methods and Models in Automation and Robotics MMAR 2004, Międzyzdroje, Poland, pp. 1355-1360.

Mayne D., Rawlings J., Rao C. and Scokaert P. (2000). Constrained model predictive control: Stability and optimality, Automatica 36(6): 789-814.

Mignone D., Ferrari-Trecate G. and Morari M. (2000). Stability and stabilization of piecewise affine and hybrid systems: An LMI approach, Proceedings of the 39-th IEEE Conference on Decision and Control, Sydney, Australia, pp. 504509.

Newell R. and Lee P. (1989). Applied Process Control - A Case Study, Prentice Hall, London.

Qin S. and Badgwell T. (2003). A survey of industrial model predictive control technology, Control Engineering Practice 11(7): 733-764.

Richter J., Schlage T. and Lunze J. (2007). Control reconfiguration of a thermofluid process by means of a virtual actuator, IET Control Theory and Applications 1(6): 1606-1620.

Rossiter J. (2003). Model-Based Predictive Control, CRC Press, Boca Raton, FL.

Staroswiecki M., Yang H. and Jiang B. (2007). Progressive accommodation of parametric faults in linear quadratic control, Automatica 43(12): 2070-2076. 
Tatjewski P. (2007). Advanced Control of Industrial Processes; Structures and Algorithms, Springer-Verlag, London.

Tondell P., Johansen T. and Bemporad A. (2003). An algorithm for multiparametric quadratic programming and explicit MPC solutions, Automatica 39(3): 489-497.

Tvrzska de Gouvea M. and Odloak D. (1998). One-layer real time optimization of LPG production in the FCC unit: Procedure, advantages and disadvantages, Computers and Chemical Engineering 22(Supplement 1): S191-S198.

Venkatasubramanian V., Rengaswamy R., Yin K. and Kavuri, S. (2003). A review of process fault detection and diagnosis, Computers and Chemical Engineering 27(3): 293-346.

Yen G. and Ho L. (2003). Online multiple-model-based fault diagnosis and accommodation, IEEE Transactions on Industrial Electronics 50(2): 296-312.
Zanin A., de Gouvea M. T. and Odloak D. (2000). Industrial implementation of a real-time optimization strategy for maximizing production of LPG in a FCC unit, Computers and Chemical Engineering 24(2-7): 525-531.

Zanin A., de Gouvea M. T. and Odloak D. (2002). Integrating real-time optimization into the model predictive controller of the FCC system, Control Engineering Practice 10(8): 819-831.

Zhang Y. (2007). Active fault-tolerant control systems: Integration of fault diagnosis and reconfigurable control, in J. Korbicz, K. Patan and M. Kowal (Eds), Fault Diagnosis and Fault Tolerant Control, Academic Publishing House EXIT, Warsaw, pp. 21-41.

Received: 20 December 2007 
